\title{
Second-order perturbation theory for fractional occupation systems: Applications to ionization potential and electron affinity calculations
}

\author{
Neil Qiang Su, Xin $\mathrm{Xu}^{*}$ \\ Collaborative Innovation Center of Chemistry for Energy Materials, Shanghai Key Laboratory of \\ Molecular Catalysis and Innovative Materials, MOE Laboratory for Computational Physical \\ Science, Department of Chemistry, Fudan University, Shanghai, 200433, China
}

(1) Data, drawn in Figure 8, for numerical and analytic derivatives of the MP2 energy with respective to occupation numbers are provided below. They correspond to $1 \mathrm{~s}, 2 \mathrm{~s}$, 2p, and 3s of Ne atom, respectively. As was recommended in Ref 4 (i.e., Beste, A.; Vázquez-Mayagoitia, Á.; Ortiz, J. V. J. Chem. Phys. 2013, 138, 074101.), the basis set for occupied orbitals (1s, 2s, and 2 p) is a completely uncontracted cc-pVTZ basis set with the spherical angular functions, while the basis set for unoccupied orbital ( $3 \mathrm{~s})$ is the cc-pVTZ plus Dunning-Hay double Rydberg basis set with the spherical angular functions. For benchmarking purpose, the variable, tol2e in NWChem, was set very tightly to be $1 \mathrm{E}-16$ and the SCF energies were always converged to be less than $1 \mathrm{E}-11$ au. At the points of fractional occupations, finite difference calculations were carried out using a formula of $\frac{E_{M P 2}\left(n_{t}+\Delta n_{t} / 2\right)-E_{M P 2}\left(n_{t}-\Delta n_{t} / 2\right)}{\Delta n_{t}}$ with $\Delta n_{t}=0.001$. At the integer points of $n_{t}=0.0$ or $n_{t}=1.0$, a less accurate formula of $\frac{E_{M P 2}\left(n_{t}+\Delta n_{t}\right)-E_{M P 2}\left(n_{t}\right)}{\Delta n_{t}}$ or $\frac{E_{M P 2}\left(n_{t}\right)-E_{M P 2}\left(n_{t}-\Delta n_{t}\right)}{\Delta n_{t}}$ can only be applied.

$\begin{array}{llll}1 \text { s occ. } & \text { Finite difference }(\mathrm{eV}) & \text { Analytic }(\mathrm{eV}) & \text { Deviation }(\mathrm{eV}) \\ 0.0 & -877.429504 & -877.432675 & -0.003171 \\ 0.1 & -876.682982 & -876.682983 & -0.000001 \\ 0.2 & -875.686063 & -875.686059 & 0.000004 \\ 0.3 & -874.422850 & -874.422848 & 0.000002 \\ 0.4 & -872.879517 & -872.879517 & 0.000000 \\ 0.5 & -871.049190 & -871.049188 & 0.000002 \\ 0.6 & -868.933555 & -868.933556 & -0.000001 \\ 0.7 & -866.544180 & -866.544181 & -0.000001 \\ 0.8 & -863.903200 & -863.903201 & -0.000001 \\ 0.9 & -861.042925 & -861.042925 & -0.000001\end{array}$




\begin{tabular}{|c|c|c|c|}
\hline 1.0 & -858.018063 & -858.002475 & 0.015588 \\
\hline $2 \mathrm{~s}$ occ. & Finite difference $(\mathrm{eV})$ & Analytic (eV) & Deviation $(\mathrm{eV})$ \\
\hline 0.0 & -49.862124 & -49.862157 & -0.000033 \\
\hline 0.1 & -49.836208 & -49.836201 & 0.000007 \\
\hline 0.2 & -49.771071 & -49.771060 & 0.000011 \\
\hline 0.3 & -49.661342 & -49.661333 & 0.000009 \\
\hline 0.4 & -49.500931 & -49.500935 & -0.000004 \\
\hline 0.5 & -49.283040 & -49.283031 & 0.000009 \\
\hline 0.6 & -48.999961 & -48.999956 & 0.000005 \\
\hline 0.7 & -48.643139 & -48.643134 & 0.000005 \\
\hline 0.8 & -48.202987 & -48.202984 & 0.000003 \\
\hline 0.9 & -47.668769 & -47.668773 & -0.000004 \\
\hline 1.0 & -47.031850 & -47.028376 & 0.003474 \\
\hline 2 p occ. & Finite difference $(\mathrm{eV})$ & Analytic (eV) & Deviation (eV) \\
\hline 0.0 & -21.710950 & -21.710274 & 0.000676 \\
\hline 0.1 & -21.826915 & -21.826999 & -0.000084 \\
\hline 0.2 & -21.906604 & -21.906654 & -0.000050 \\
\hline 0.3 & -21.941546 & -21.941522 & 0.000024 \\
\hline 0.4 & -21.922859 & -21.922746 & 0.000113 \\
\hline 0.5 & -21.840188 & -21.840176 & 0.000012 \\
\hline 0.6 & -21.682381 & -21.682364 & 0.000017 \\
\hline 0.7 & -21.436353 & -21.436486 & -0.000133 \\
\hline 0.8 & -21.088418 & -21.088413 & 0.000005 \\
\hline 0.9 & -20.622895 & -20.622836 & 0.000059 \\
\hline 1.0 & -20.026960 & -20.023611 & 0.003349 \\
\hline $3 \mathrm{~s}$ occ. & Finite difference $(\mathrm{eV})$ & Analytic (eV) & Deviation (eV) \\
\hline 0.0 & 0.282805 & 0.282792 & -0.000013 \\
\hline 0.1 & 0.282854 & 0.282848 & -0.000006 \\
\hline 0.2 & 0.282893 & 0.282893 & 0.000000 \\
\hline 0.3 & 0.282937 & 0.282937 & 0.000000 \\
\hline 0.4 & 0.282982 & 0.282980 & -0.000002 \\
\hline 0.5 & 0.283024 & 0.283023 & -0.000001 \\
\hline 0.6 & 0.283066 & 0.283065 & -0.000001 \\
\hline 0.7 & 0.283107 & 0.283107 & 0.000000 \\
\hline 0.8 & 0.283149 & 0.283148 & -0.000001 \\
\hline 0.9 & 0.283190 & 0.283189 & -0.000001 \\
\hline 1.0 & 0.283230 & 0.283230 & 0.000000 \\
\hline
\end{tabular}

The residual differences between the results from finite difference and analytic calculations shall come from numeric errors. Nearly full agreement can be established when $\Delta n_{t}$ is set to be 0.000001 and the SCF energies are converged to be less than 
1E-13. For example, by using $\frac{E_{M P 2}\left(n_{t}+0.000001\right)-E_{M P 2}\left(n_{t}\right)}{0.000001}$ and $\frac{E_{M P 2}\left(n_{t}\right)-E_{M P 2}\left(n_{t}-0.000001\right)}{0.000001}$ to calculate the finite difference results at the points $n_{t}=0.0$ and $n_{t}=1.0$, respectively, for $1 \mathrm{~s}$, we have -877.432668 and $-858.002480 \mathrm{eV}$, as compared to the analytical results of -877.432675 and $-858.002475 \mathrm{eV}$, respectively. The deviations from analytic results are reduced from -0.003171 and 0.015588 to -0.000007 and $0.000005 \mathrm{eV}$, respectively.

(2) Geometries optimized at the MP2(full)/cc-pVTZ level with the spherical angular functions.

$\begin{array}{cccc}\mathrm{F}_{2} & & & \\ 01 & & & \\ \mathrm{~F} & 0.00000000 & 0.00000000 & -0.69790814 \\ \mathrm{~F} & 0.00000000 & 0.00000000 & 0.69790814 \\ & & & \\ \mathrm{OH} & & & \\ 02 & & & \\ \mathrm{O} & 0.00000000 & 0.00000000 & -0.10583476 \\ \mathrm{H} & 0.00000000 & 0.00000000 & 0.86004588 \\ & & & \\ \mathrm{NH}_{2} & & & \\ 02 & & & -0.14113983 \\ \mathrm{~N} & 0.00000000 & 0.00000000 & 0.49370325 \\ \mathrm{H} & 0.00000000 & 0.79719018 & 0.49370325 \\ \mathrm{H} & 0.00000000 & -0.79719018 & \\ & & & \\ \mathrm{CH} & & & \\ 02 & & & 0.000000000 \\ \mathrm{C} & 0.00000000 & 0.00000000 & 0.00000000000 \\ \mathrm{H} & 1.07084373 & 0.00000000 & 0.00000000 \\ \mathrm{H} & -0.53542196 & -0.92739188 & \\ \mathrm{H} & -0.53542196 & 0.92739188 & \\ & & & \\ \mathrm{CN} & & & \\ 02 & & & \\ \mathrm{C} & 0.00000000 & 0.00000000 & \\ \mathrm{~N} & 0.00000000 & 0.00000000 & 0.009833\end{array}$


$\mathrm{O}_{2}$

03

$\begin{array}{llll}\mathrm{O} & 0.00000000 & 0.00000000 & -0.61018739\end{array}$

$\begin{array}{llll}\text { O } & 0.00000000 & 0.00000000 & 0.61018739\end{array}$

$\begin{array}{cccc}\mathrm{H}_{2} \mathrm{O} & & & \\ 01 & & & \\ \mathrm{O} & 0.00000000 & 0.00000000 & 0.11792448 \\ \mathrm{H} & 0.00000000 & 0.75280146 & -0.47381746 \\ \mathrm{H} & 0.00000000 & -0.75280146 & -0.47381746\end{array}$

$\mathrm{CH}_{2} \mathrm{O}$

01

$\begin{array}{llll}\text { C } & 0.00000000 & 0.00000000 & -0.52945193\end{array}$

$\begin{array}{llll}\mathrm{O} & 0.00000000 & 0.00000000 & 0.67799661\end{array}$

$\begin{array}{llll}\mathrm{H} & 0.93057144 & 0.00000000 & -1.10890584\end{array}$

$\begin{array}{llll}\mathrm{H} & -0.93057144 & 0.00000000 & -1.10890584\end{array}$

$\mathrm{CH}_{4}$

01

$\begin{array}{llll}\text { C } & 0.00000000 & 0.00000000 & 0.00000000\end{array}$

$\begin{array}{llll}\mathrm{H} & 0.00000000 & 0.000000000 & 1.08243940\end{array}$

$\begin{array}{llll}\mathrm{H} & 1.02053379 & 0.00000000 & -0.36081301\end{array}$

$\begin{array}{llll}\mathrm{H} & -0.51026668 & 0.88380808 & -0.36081320\end{array}$

$\begin{array}{llll}\mathrm{H} & -0.51026668 & -0.88380808 & -0.36081320\end{array}$

$\mathrm{NH}_{3}$

01

$\begin{array}{llll}\mathrm{N} & 0.00000000 & 0.00000000 & 0.11428516\end{array}$

$\begin{array}{llll}\mathrm{H} & 0.00000000 & 0.93367418 & -0.26649106\end{array}$

$\mathrm{H} \quad 0.80858561 \quad-0.46683707 \quad-0.26649105$

$\mathrm{H} \quad-0.80858561 \quad-0.46683707 \quad-0.26649105$

N2

01

$\begin{array}{llll}\mathrm{N} & 0.00000000 & 0.00000000 & 0.55509301\end{array}$

$\begin{array}{llll}\mathrm{N} & 0.00000000 & 0.00000000 & -0.55509301\end{array}$ 